\title{
An Economic Evaluation Supported by Qualitative Data About the Patient Concerns Inventory (PCl) versus Standard Treatment Pathway in the Management of Patients with Head and Neck Cancer
}

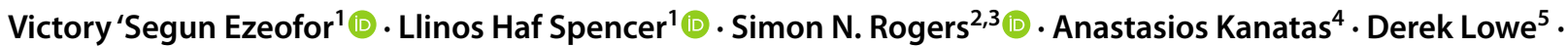 \\ Cherith J. Semple ${ }^{6,7}$ (D) Jeffrey R. Hanna ${ }^{8} \cdot$ Seow Tien Yeo ${ }^{1} \cdot$ Rhiannon Tudor Edwards $^{1}$
}

Accepted: 16 December 2021 / Published online: 31 January 2022

(c) The Author(s) 2022

\begin{abstract}
Background The head and neck cancer (HNC) Patient Concerns Inventory (PCI) is a condition-specific prompt list that allows patients to raise concerns to cancer consultants that otherwise might be overlooked.

Objective This is the first economic evaluation of the PCI in patients with $\mathrm{HNC}$ investigating the costs and effects to the health service of not prioritising certain treatment pathways in addition to the primary cancer pathway. Additional costs can be accrued due to delayed referral to other appropriate services, e.g. hospital dentist. Economic evidence could influence future policy direction in this area globally.

Methods Alongside a 3-year clustered randomised controlled trial, an economic evaluation was undertaken with Client Service Receipt Inventory data collected at three different time points (baseline and 6 and 12 months post-baseline). Patients were identified by a multidisciplinary team at the trial clinics. This economic analysis compared the PCI intervention versus the non-PCI treatment pathway. A deterministic and probabilistic sensitivity analysis was conducted to investigate the cost per quality-adjusted life-year (QALY) gain of the PCI versus non-PCI intervention treatment pathways. Qualitative data were also collected from seven consultants to triangulate findings from the economic evaluation.

Results The analysis used data from 191 patients (66\% of the full trial sample). The PCI inventory was low cost, at just over $£ 13$ per participant. The PCI intervention was cost effective and also cost saving, with an incremental cost difference of $£ 295.91$ over the 12-month follow-up period. The QALY values were higher in the PCI intervention strategy, with a value of 0.79 , whereas the non-PCI group had a value of 0.76 , thus the PCI intervention was dominant. The sensitivity analysis showed that, at a willingness-to-pay threshold of $£ 20,000$ per QALY gained, the probability of being cost effective was 0.85 (95\% confidence interval [CI] 0.80-0.83). Qualitative results showed that consultants using the PCI reported an enhanced awareness of patients' overall post-treatment needs.

Discussion The PCI provided an effective means to conduct clinical consultations by avoiding unnecessary healthcare costs and focussing on aspects of care most important to patients. The cost per QALY gain was within the National Institute for Health and Care Excellence guideline threshold. The economic evaluation showed that the PCI intervention strategy was dominant and therefore cost saving to the national health service (NHS) and was more effective in terms of treatment.

Conclusion The PCI appears to be a low-cost intervention that generates a cost-effective benefit to patients from a NHS perspective if rolled out as part of routine care. Qualitative evidence has shown that the use of the PCI is supported by consultants in routine practice.
\end{abstract}

Trial Registration Clinical Trials Identifier: NCT03086629.

Victory 'Segun Ezeofor

v.ezeofor@bangor.ac.uk

Extended author information available on the last page of the article 


\section{Key Points for Decision Makers}

This study is the first economic evaluation that compares the Patient Concerns Inventory (PCI) with the standard treatment pathways.

The study presents the cost benefits of using the PCI for patient referral to the appropriate consultant(s) in head and neck cancer (HNC) treatment.

Evidence of the impact of the costs and effects of the PCI treatment pathway is presented to enable healthcare providers make informed decisions regarding $\mathrm{HNC}$ treatment.

\section{Background}

Head and neck cancers (HNC), including mouth, throat and voice box cancer, account for around 12,000 new cancer diagnoses in the UK each year [1]. Surgery, chemotherapy and radiotherapy are standard treatments for HNC but can cause many other issues such as changes in facial appearance and difficulties with chewing, speech and swallowing. These in turn can have a detrimental impact on functioning and emotional wellbeing, social interaction and quality of life (QOL) [2]. Time constraints in oncology clinics mean that patients with HNC do not always have the opportunity to raise issues of concern in their follow-up clinical consultations [3, 4].

The Questionnaire Prompt Lists is a constructive communication tool that enhances patient participation in oncology consultations [5]. It allows patients to raise their concerns and help optimise time utilisation during consultations [6, 7]. The fear of cancer recurrence is common, but patients with multiple emotional concerns need additional support at various points during and after treatment [8]. This could be challenging for the clinical team because of the time constraints imposed upon them [8].

The HNC Patient Concerns Inventory (PCI) was first published in 2009 by the research team [9] and is a conditionspecific prompt list that allows patients to raise concerns that otherwise might be overlooked [5]. A recent systematic review and content comparison of self-report measures of unmet needs used in patients with HNC favoured the PCI over 13 other tools [10].

The PCI consists of 56 clinical items and has been used by patients in outpatient clinics before seeing their consultant. The list serves to guide the outpatient consultation and covers a range of symptoms and potential problems patients may face after treatment. It has been shown to be feasible in routine consultations $[11,12]$ and for wider adoption across a cancer network [13]. The PCI is freely available online (http://www.patient-concerns-inventory.co.uk). The PCI supports several national initiatives such as the national health service (NHS) Holistic Needs Assessment for People with Cancer [14] and is set in the context of the national debate on how to bring about more person-centred care and the National Survivorship Initiative [15].

The 3-year National Institute for Health Research-funded study by Rogers et al. [16]. ClinicalTrials.gov identifier, NCT03086629) investigated the use of the PCI in a randomised controlled trial (RCT) across multiple consultants at two locations in the North of England, with the aim of reporting the a priori outcomes of the RCT, specifically overall QOL, social-emotional dysfunction and distress following repeated use of the PCI-based summary sheet after a 1 -year period. To get a standardised cost solely for the treatment and treatment pathway, costs were collected using the Client Services Receipt Inventory (CSRI) [17]. The CSRI was used to record participants' contacts with primary and secondary health and social care services.

As part of the economic evaluation, this study was undertaken from a UK NHS perspective with costs derived from the CSRI. This was done alongside the PCI HNC RCT, with the generic health-related QOL (HRQOL) measure EQ5D-5L [18] used for the quality-adjusted life-year (QALY) calculation, to explore the cost effectiveness of the PCI in the treatment of patients with HNC. A rapid review was conducted, and no evidence was found of a previous health economic analysis of the PCI intervention. Hence, this economic evaluation study will add an economic foundation by contributing a different dimension to the published literature about PCI interventions. Future studies might also consider including societal costs and other costs where necessary to give a more global overview.

\section{Methods and Design}

This economic evaluation was conducted alongside a 3-year clustered RCT. CSRI data were collected at three different time points (baseline and 6 and 12 months post-baseline). At the cluster level, there was similarity between trial groups in the number of patients, ages, and consultation time, but there were differences between the type of patient (tumour location and staging) and treatment administered (see Rogers et al. [16] for more details). In this trial, there were nine consultants at the Liverpool site and six consultants at the Leeds site. Eight consultants were assigned to the PCI group and seven to the non-PCI group.

The PCI is a condition-specific prompt list including 56 clinical items that allows patients to raise concerns to cancer 
consultants that otherwise might be overlooked during their consultation (an example of the PCI inventory is shown in the electronic supplementary material [ESM]-1). This health economic analysis investigates the cost effectiveness of the HRQOL between two groups of patients, one of which received the PCI as part of their treatment pathway. In-depth details about the PCI trial are published elsewhere [16]; in this paper, we report on the economic evaluation along with the nested consultant qualitative interviews. In the original PCI trial, a total of 254 patients were invited to take part in the intervention group, and 257 were invited to take part in the non-PCI group. Following refusals and withdrawals, there were 140 in the PCI group and 148 in the non-PCI group. Data were collected from both groups at baseline and twice during the trial period (at 6 and 12 months from baseline). The main results from the clinical effectiveness study [16] are presented in Appendix 1.

\subsection{Qualitative Interviews Methodology}

Qualitative methods are increasingly being used alongside trials and health economic analyses to complement the standard statistical analysis with further insightful information from stakeholders [19, 20]. In the PCI RCT [16], face-to-face, semi-structured interviews were carried out with consultants by the third author of this paper (SNR), who is a subject expert in HNC and HRQOL aided by a topic guide (see ESM-2). Consultants who delivered the PCI intervention were asked about how they were trained to use the PCI, perceived usefulness of the PCI, using the PCI in practice, perceived barriers in using the PCI, and any other issues relating to using the PCI. The development of the topic guide was directed by expert opinion from the research team and literature surrounding process evaluation for intervention development. Using a convenience sampling approach, all the consultants $(n=8)$ in the PCI arm of the trial were approached initially by email to ascertain whether they would be interested and willing to participate; only one declined to take part. Interviews took place within the healthcare setting, were audio-recorded and lasted between 20 and $30 \mathrm{~min}$. Audio recordings were transcribed verbatim and analysed using the theoretical domains framework lens [21]. Inductive data analysis took place using the six-step approach to reflexive thematic analysis of qualitative data. Initially, a qualitative researcher (CJS) read and reread the transcripts. As an inductive process, codes were developed and collated (CJS), identifying initial themes. To ensure rigour and credibility, the codes and themes were independently analysed by another experienced qualitative researcher (JRH).

\subsection{Economic Evaluation Methods}

\subsubsection{About the Patient Concerns Inventory (PCl) Randomised Controlled Trial}

The HNC PCI RCT was conducted at hospitals in Leeds and Liverpool, England. Baseline clinics ran from April 2017 to October 2019 with 140 patients in the PCI intervention arm and 148 non-PCI patients. The RCT is described in more detail elsewhere [16]. In the UK, most healthcare costs are covered by the NHS, including costs such as treatment costs, administrative and monitoring costs, health resource use costs and cost of managing adverse effects. Therefore, this economic evaluation was conducted from an NHS perspective, as this trial was conducted in the UK.

The CSRI data were collected by the study research nurses, as close to 6 and 12 months post-baseline as possible; they were usually collected at the clinic along with other trial data but occasionally were collected separately by telephone from study participants. Data from the clinical trial are synthesised to deduce variable values that were inputted into the health economic evaluation. Table 1 shows the characteristics of the economic analysis sample. The hot deck method $[22,23]$ was applied to handle missing data, and the results are compared with analysis of those with complete data. The hot deck method replaces missing data with data from the dataset that have similar properties; this gives a reasonable distribution to the data instead of clogging the mean value. When the results were similar, only patients who completed the baseline, intermediate follow-up and final follow-up were included in the sensitivity analysis. This was done to avoid bias by imputation or other methods of computing missing variables [30].

The methods used in this economic evaluation can be replicated globally to investigate other costs, such as societal costs, that are not captured in the UK NHS services costs.

\subsubsection{Cost Data}

In preparing the costs involved in the study, service use data were used to provide a comprehensive treatment cost per patient. Most observed costs were linked to consultant time as only a few patients with HNC needed additional treatments during the trial period. The costs for contact with healthcare professionals involved in this study are presented in Tables 2 and 3. The costs of services used were retrieved from published literature [24] and NHS reference costs [25] for the year 2020. Resource use data were collected using the CSRI tool [17], and the resource use for the PCI and non-PCI groups is presented in Table 2. 
Table 1 Characteristics of the economic analysis sample

\begin{tabular}{|c|c|c|}
\hline Characteristic & PCI & Non-PCI \\
\hline Sample size & 90 & 101 \\
\hline Mean age, years & $63.15 \pm 10.79$ & $60.41 \pm 10.79$ \\
\hline Male & $56(62)$ & $75(74)$ \\
\hline \multicolumn{3}{|l|}{ Ethnicity } \\
\hline White British & 89 (99) & $96(95)$ \\
\hline Other & $1(1)$ & $5(5)$ \\
\hline \multicolumn{3}{|l|}{ In employment } \\
\hline Yes & $37(41)$ & $30(30)$ \\
\hline No & $50(56)$ & $70(69)$ \\
\hline Not known & $3(3)$ & $1(1)$ \\
\hline \multicolumn{3}{|l|}{ In receipt of benefit } \\
\hline None & $57(63)$ & $60(59)$ \\
\hline Some & $28(31)$ & $36(36)$ \\
\hline Not known & 5 & 5 \\
\hline \multicolumn{3}{|l|}{ Tobacco use } \\
\hline Current user & $10(11)$ & $12(12)$ \\
\hline Former user & $56(62)$ & $54(53)$ \\
\hline Never used & $22(24)$ & $32(32)$ \\
\hline Not known & $2(3)$ & $3(3)$ \\
\hline \multicolumn{3}{|c|}{$\begin{array}{l}\text { Education and skills (small area } \\
\text { IMD summary deciles) }\end{array}$} \\
\hline 1 & $12(13)$ & $22(22)$ \\
\hline 2 & 17 (19) & $8(8)$ \\
\hline 3 & $5(6)$ & $6(6)$ \\
\hline 4 & $5(6)$ & $7(7)$ \\
\hline 5 & $8(9)$ & $9(9)$ \\
\hline 6 & $7(8)$ & $7(7)$ \\
\hline 7 & $5(6)$ & $10(10)$ \\
\hline 8 & $9(10)$ & $11(11)$ \\
\hline 9 & $15(17)$ & $8(8)$ \\
\hline 10 & $7(8)$ & $13(13)$ \\
\hline \multicolumn{3}{|l|}{ EQ-5D-5L TTO } \\
\hline Baseline & $0.78 \pm 0.17$ & $0.75 \pm 0.21$ \\
\hline Intermediate follow-up ${ }^{a}$ & $0.79 \pm 0.21$ & $0.76 \pm 0.22$ \\
\hline Final follow-up ${ }^{b}$ & $0.80 \pm 0.21$ & $0.76 \pm 0.23$ \\
\hline \multicolumn{3}{|l|}{ EQ-5D-5L VAS } \\
\hline Baseline & $73.38 \pm 17.30$ & $71.92 \pm 19.24$ \\
\hline Intermediate follow-up & $75.82 \pm 17.96$ & $73.66 \pm 21.86$ \\
\hline Final follow-up & $76.02 \pm 18.98$ & $73.90 \pm 20.56$ \\
\hline \multicolumn{3}{|l|}{ Consultation time, minutes } \\
\hline Baseline & $12.83(6.91)$ & $10.68(5.89)$ \\
\hline Intermediate follow-up & $9.74(4.63)$ & $9.63(4.02)$ \\
\hline Final follow-up & $9.19(4.28)$ & $9.90(6.26)$ \\
\hline
\end{tabular}

Data are presented as mean \pm standard deviation or $\mathrm{n}(\%)$ unless otherwise indicated

IMD Index of Multiple Deprivation, PCI Patient Concerns Inventory, TTO time-trade-off, VAS visual analogue scale

${ }^{a}$ Data were obtained at around 6 months from baseline

${ }^{b}$ Data were obtained around 12 months from baseline

\subsubsection{Costs of the $\mathrm{PCl}$ Intervention}

All costs about the intervention strategy are classified under two basic categories: training cost and intervention cost. The training cost indicates the cost that would be accrued if this trial were to be replicated elsewhere. The intervention cost is composed of all added costs accrued when patients receive this particular intervention. A breakdown of these costs is summarised in Tables 2 and 3. Table 3 shows the micro-costing of the intervention [26], and the additional cost of about $£ 13$ per participant for training is included in the cost-effectiveness analysis. The total cost of training the eight PCI consultants was about $£ 1048$.

Clinical-effectiveness results of the PCI intervention are published elsewhere [12] (see Appendix 1 for the summary) and indicated that length of the consultation time had a tendency to reduce with subsequent use of the PCI in comparison with the non-PCI group.

\subsection{Cost Effectiveness}

The cost-effectiveness analysis was used to investigate the costs and health outcomes of patients with HNC using the PCI or not using the PCI intervention strategy. The EQ5D-5L measure is a self-reported QOL measure with five levels of responses [18]. The EQ-5D-5L data were collected at three time points (baseline, intermediate follow-up at around 6 months and final follow-up at around 12 months). The EQ-5D-5L scores were cross walked, meaning that the responses to the EQ-5D-5L were linked to the EQ-5D-3L value sets [27] as recommended by the National Institute for Care and Excellence (NICE) [28]; mean scores are presented in Table 1. The HRQoL obtained from the EQ-5D are computed using the area under the curve (AUC) methodology. The AUC equates to the QALY value for each patient as the average EQ-5D value over the period of trial.

\subsubsection{Primary Health Economics Outcome Measure}

The primary outcome measure was the incremental costeffectiveness ratio (ICER) computed as the cost per QALY gain. The ICER value between the PCI and the non-PCI group was computed as a ratio of the difference in costs and QALYs over a 12-month horizon, so no discounting was required for the costs and QALYs.

\subsubsection{Sensitivity Analysis}

A non-parametric bootstrapping method was applied to estimate the outcome by sampling with replacement from the original trial data [29] as a check of the robustness of the analysis. The probabilistic sensitivity analyses were used to evaluate the impact of uncertainty across all the parameters 
Table 2 Average \pm standard deviation healthcare cost (£) obtained from the resource use (CSRI) amongst patients in the PCI trial

\begin{tabular}{|c|c|c|}
\hline Description & PCI & Non-PCI \\
\hline HNC consultant F2F & $735.96 \pm 297.61$ & $879.41 \pm 356.77$ \\
\hline HNC consultant non-F2F & $0.00 \pm 0.00$ & $1.07 \pm 10.75$ \\
\hline $\mathrm{HNC}$ oncologist $\mathrm{F} 2 \mathrm{~F}$ & $166.22 \pm 244.46$ & $193.90 \pm 267.44$ \\
\hline $\mathrm{HNC}$ registrar $\mathrm{F} 2 \mathrm{~F}$ & $12.60 \pm 40.11$ & $13.63 \pm 39.80$ \\
\hline HNC CNS F2F & $4.28 \pm 16.95$ & $14.70 \pm 47.88$ \\
\hline HNC CNS non-F2F & $1.00 \pm 6.67$ & $0.89 \pm 6.30$ \\
\hline $\mathrm{HNC}$ nurses F2F & $8.39 \pm 33.60$ & $17.75 \pm 142.26$ \\
\hline $\mathrm{HNC}$ nurses non-F2F & $0.59 \pm 5.59$ & $2.10 \pm 21.10$ \\
\hline Hospital dental hygienist F2F & $1.77 \pm 6.11$ & $3.37 \pm 13.77$ \\
\hline Hospital dentist F2F & $99.01 \pm 278.81$ & $75.06 \pm 206.40$ \\
\hline Oral rehabilitation team F2F & $17.90 \pm 91.79$ & $35.09 \pm 175.90$ \\
\hline Oral rehabilitation team non-F2F & $1.52 \pm 14.44$ & $0.00 \pm 0.00$ \\
\hline Dietician F2F & $32.41 \pm 83.46$ & $39.71 \pm 144.94$ \\
\hline Physiotherapist F2F & $15.89 \pm 65.05$ & $22.33 \pm 74.27$ \\
\hline SLT F2F & $64.71 \pm 149.19$ & $55.60 \pm 138.41$ \\
\hline SLT non-F2F & $3.50 \pm 15.42$ & $0.89 \pm 6.30$ \\
\hline Audiologist F2F & $24.00 \pm 68.26$ & $6.42 \pm 29.86$ \\
\hline Audiologist non-F2F & $0.61 \pm 5.80$ & $0.00 \pm 0.00$ \\
\hline Medical inpatient ward No. of nights & $0.00 \pm 0.00$ & $3.25 \pm 32.64$ \\
\hline $\mathrm{X}$-rays & $16.00 \pm 39.51$ & $16.93 \pm 50.45$ \\
\hline Magnetic resonance imaging & $25.00 \pm 80.98$ & $23.17 \pm 51.94$ \\
\hline CT scan & $15.00 \pm 45.25$ & $11.58 \pm 39.57$ \\
\hline PET scan & $0.00 \pm 0.00$ & $0.89 \pm 8.96$ \\
\hline Biopsy & $86.57 \pm 229.65$ & $66.12 \pm 177.12$ \\
\hline A\&E attendance & $1.88 \pm 17.88$ & $10.08 \pm 52.67$ \\
\hline A\&E taken by ambulance to hospital A\&E & $0.00 \pm 0.00$ & $1.03 \pm 10.34$ \\
\hline A\&E seen by consultant & $1.20 \pm 11.38$ & $4.28 \pm 21.17$ \\
\hline A\&E seen by non-consultant & $0.00 \pm 0.00$ & $2.14 \pm 15.12$ \\
\hline GP surgery & $49.46 \pm 129.77$ & $81.86 \pm 218.71$ \\
\hline GP phone or email & $0.00 \pm 0.00$ & $1.09 \pm 10.95$ \\
\hline GP practice nurse surgery & $0.00 \pm 0.00$ & $1.86 \pm 14.75$ \\
\hline District nurse surgery & $8.00 \pm 75.90$ & $0.71 \pm 7.16$ \\
\hline District nurse home & $0.00 \pm 0.00$ & $32.44 \pm 325.97$ \\
\hline District nurse phone or email & $0.00 \pm 0.00$ & $0.91 \pm 9.15$ \\
\hline Community nurse specialist surgery & $0.61 \pm 5.80$ & $25.05 \pm 181.34$ \\
\hline Dentist surgery & $131.52 \pm 170.92$ & $158.02 \pm 204.07$ \\
\hline Dentist phone or email & $2.31 \pm 21.93$ & $0.00 \pm 0.00$ \\
\hline Community physiotherapist surgery & $0.38 \pm 3.58$ & $1.68 \pm 12.14$ \\
\hline Community SLT surgery & $7.47 \pm 54.28$ & $2.85 \pm 21.27$ \\
\hline Community SLT phone or email & $0.50 \pm 4.74$ & $0.00 \pm 0.00$ \\
\hline Community occupational health therapist surgery & $0.00 \pm 0.00$ & $0.64 \pm 6.47$ \\
\hline Community audiologist surgery & $0.96 \pm 9.07$ & $0.00 \pm 0.00$ \\
\hline Clinical psychologist surgery & $5.67 \pm 39.89$ & $30.30 \pm 215.57$ \\
\hline
\end{tabular}

$A \& E$ accident and emergency, $C N S$ clinical nurse specialist, $C S R I$ Client Service Receipt Inventory, $C T$ computed tomography, F2F face to face, $H N C$ head and neck cancer, PCI Patient Concerns Inventory, PET positron emission tomography, $S L T$ speech and language therapist simultaneously. To reflect the uncertainty in the mean costs and effects, an ICER scatterplot of the 2000 bootstrapped incremental cost and effect is presented on an ICER plane.
Values from the ICER iterations were used to develop the cost-effectiveness analysis curve (CEAC) for various willingness-to-pay (WTP) thresholds. The CEAC represents the 
Table 3 Micro-costing of the intervention

\begin{tabular}{|c|c|c|}
\hline Item & Description & Total cost \\
\hline \multicolumn{3}{|l|}{ Training costs } \\
\hline \multicolumn{3}{|l|}{ Leeds } \\
\hline Consultant trainer & $£ 143 / \mathrm{h}$ (one trainer) & \\
\hline Duration of training & Group session of $1 \mathrm{~h}$ & $£ 143.00$ \\
\hline Number of trainees & Three trainees in a group & \\
\hline Consultants (trainee) & $£ 143 / \mathrm{h}$ & $£ 429.00$ \\
\hline Subtotal & & $£ 572.00$ \\
\hline \multicolumn{3}{|l|}{ Aintree, Liverpool } \\
\hline Consultant trainer & $£ 143 / \mathrm{h}$ (one trainer) & \\
\hline Duration of training & 20 minutes, one to one & \\
\hline Aintree, Liverpool & Five trainees, trained individually & $£ 238.33$ \\
\hline Consultants (trainee) & $£ 47.67$ for 20 minutes $(£ 143 / \mathrm{h})$ & $£ 238.33$ \\
\hline Subtotal & & $£ 476.66$ \\
\hline \multicolumn{3}{|l|}{ General cost } \\
\hline Travel & $£ 0^{\mathrm{a}}$ & \\
\hline Venue & $£ 0^{\mathrm{a}}$ & \\
\hline Total cost (training) & & $£ 1048.67$ \\
\hline \multicolumn{3}{|l|}{ Intervention cost } \\
\hline Volunteer cost & $\begin{array}{l}£ 47-55 \text { (mean cost for GP nurse or CNS for } 15 \\
\text { minutes) }\end{array}$ & $£ 12.75$ per participant \\
\hline Print out (black \& white) & $£ 0.10$ per print (three print outs required) & $£ 0.30$ per participant \\
\hline Online PCI form & $£ 0$ & $£ 0$ \\
\hline Total cost (intervention) & & $£ 13.05$ per participant \\
\hline
\end{tabular}

CNS clinical nurse specialist, GP general practitioner, $P C I$ Patient Concerns Inventory

${ }^{a}$ Training was conducted at the trial centre (national health service sites), so no travel or venue costs were incurred probability of the intervention strategy being cost effective as compared with the control strategy at different values of payer WTP thresholds for an additional QALY gain. These values were compared with the NICE guideline threshold of $£ 20,000-30,000$ per QALY gained relative to usual care. All analyses were conducted using Microsoft Excel 365.

\section{Results}

\subsection{Base-Case Analysis}

Of the 140 participants in the intervention (PCI) group at baseline, 90 participants completed the EQ-5D-5L at all three time points (baseline, intermediate follow-up at around 6 months and final follow-up at around 12 months); of the 148 participants in the control (non-PCI) group, 101 participants had completed EQ-5D-5L data at all three time points. At baseline, the PCI group had a mean \pm standard deviation EQ-5D score of $0.78 \pm 0.17$, whereas the non-PCI group had a mean score of $0.75 \pm 0.12$. A Mann-Whitney test was conducted to investigate baseline equivalence of the average EQ-5D value between the PCI intervention group and the
non-PCI group with a $95 \%$ confidence interval (CI); the $p$ value was 0.07 , indicating that the EQ-5D values of the PCI and non-PCI groups were similar at baseline.

The cost-effectiveness result for the base-case data is shown in Table 4. The PCI intervention strategy had a lower mean cost of $£ 1542.88$, whereas the non-PCI had a mean cost of $£ 1838.79$, which had an incremental cost of $£ 295.91$ per QALY. The QALY values were higher in the PCI intervention strategy, with a value of 0.79 , whereas the non-PCI had a value of 0.76 . With a lower cost and higher effect, the PCI

Table 4 Incremental cost effectiveness of PCI intervention strategy compared with non-PCI intervention

\begin{tabular}{llllll}
\hline Intervention & Mean cost & QALYs & \multicolumn{2}{l}{ Incremental } & ICER \\
\cline { 3 - 5 } & & & Cost & QALYs & \\
\hline PCI & 1542.88 & 0.79 & -295.91 & 0.03 & Dominant \\
Non-PCI & 1838.79 & 0.76 & & & \\
\hline
\end{tabular}

Costs are presented as £

ICER incremental cost-effectiveness ratio, PCI Patient Concerns Inventory, $Q A L Y$ quality-adjusted life-years 
intervention was cost saving (dominant) to the NHS. The PCI intervention strategy, having an additional but minimal intervention cost of just over $£ 13$, was the dominant strategy for consultations in HNC treatment, as can be seen in Table 4 .

The base-case analysis of the EQ-5D-5L and cost data of each cluster of participants with complete EQ-5D-5L data at baseline, intermediate follow-up (6 months) and final followup (12 months) are shown in Table 5. The highest QALY value in the PCI group was 0.88 in cluster 3, whereas the non-PCI group had the highest value of 0.91 in cluster 5 . The lowest QALY value in the PCI group was 0.72 in cluster 5, whereas the non-PCI group had the least value of 0.72 in cluster 6 .

The results in Table 5 were compared with those in Table 6, which is the same analysis but replacing the missing data using the hot deck method (average of the mean value between the intervention group and cluster group with similar properties). The analysis for the inputted data scenario shows that cluster 3 of the PCI had the highest QALY value of 0.86 , whereas cluster 5 of the non-PCI had the highest QALY value of 0.85 . The lowest QALY value in the PCI group was cluster 5, with a value of 0.73 , and for the non-PCI group was cluster 6 , with a value of 0.69 . In terms of cost, both Tables 5 and 6 for the PCI group cluster 6 have the highest cost, with the complete case having a value of $£ 2453.52$ and the inputted scenario having a value of $£ 2184$.66. For the non-PCI group, cluster 7 had the highest value for both the complete case scenario and inputted group, with values of $£ 2669.40$ and $£ 2420.59$, respectively.

The cost effectiveness for each cluster can be analysed individually (see Appendix 2, Table 9 for all cluster analysis). As shown in Table 8 of Appendix 2 using row 1 of Table 5, the average cost of the PCI intervention strategy is $£ 1753$, whereas the non-PCI group had an average cost of $£ 1364$. The QALY values for the same cluster were 0.76 and 0.73 for the PCI and non-PCI groups, respectively. The incremental cost was $£ 390$, whereas the incremental QALY was 0.03, thus giving an ICER value of $£ 14,215$ per QALY gained. A similar result is shown in Table 8 of Appendix 2, where using row 1 of Table 6 gave

Table 5 Base-case analysis of the EQ-5D-5L, QALY (AUC) and cost for the complete case scenario

\begin{tabular}{|c|c|c|c|c|c|c|c|c|c|c|}
\hline \multirow[t]{2}{*}{ Group } & \multicolumn{5}{|l|}{ PCI } & \multicolumn{5}{|l|}{ Non-PCI } \\
\hline & EQ5D_B & EQ5D_I & EQ5D_F & QALY & Total cost (£) & EQ5D_B & EQ5D_I & EQ5D_F & QALY & Total cost (£) \\
\hline Cluster 1 & 0.7491 & 0.7367 & 0.7967 & 0.7548 & 1753.33 & 0.7817 & 0.7178 & 0.6923 & 0.7274 & 1363.84 \\
\hline Cluster 2 & 0.8631 & 0.8740 & 0.8429 & 0.8635 & 1316.45 & 0.7750 & 0.7737 & 0.7889 & 0.7778 & 2170.78 \\
\hline Cluster 3 & 0.8009 & 0.8916 & 0.9494 & 0.8834 & 1694.23 & 0.8143 & 0.6737 & 0.9223 & 0.7710 & 941.61 \\
\hline Cluster 4 & 0.7289 & 0.7722 & 0.7802 & 0.7634 & 1419.73 & 0.7440 & 0.7780 & 0.7467 & 0.7617 & 1611.00 \\
\hline Cluster 5 & 0.7879 & 0.7519 & 0.7134 & 0.7513 & 1844.01 & 0.9593 & 0.8503 & 0.9593 & 0.9048 & 1500.16 \\
\hline Cluster 6 & 0.8103 & 0.8145 & 0.8528 & 0.8231 & 2453.52 & 0.6888 & 0.7385 & 0.7240 & 0.7224 & 1996.73 \\
\hline Cluster 7 & 0.7202 & 0.7655 & 0.7336 & 0.7462 & 830.12 & 0.7713 & 0.7279 & 0.7989 & 0.7565 & 2669.40 \\
\hline Cluster 8 & 0.7834 & 0.7344 & 0.7756 & 0.7570 & 664.96 & \multicolumn{5}{|c|}{ No Cluster 8} \\
\hline
\end{tabular}

Costs are presented in GBP (£)

$A U C$ area under the curve, $E Q-5 D \_B$ EQ-5D at baseline, $E Q-5 D \_F$ EQ-5D at 12-month follow-up, EQ-5D_I EQ-5D at 6-month follow-up, $P C I$ Patient Concerns Inventory, $Q A L Y$ quality-adjusted life-years

Table 6 Base-case analysis of the EQ-5D-5L, QALY (AUC) and cost with data inputted

\begin{tabular}{|c|c|c|c|c|c|c|c|c|c|c|}
\hline \multirow[b]{2}{*}{ Group } & \multicolumn{5}{|l|}{ PCI } & \multicolumn{5}{|l|}{ Non-PCI } \\
\hline & EQ5D_B & EQ5D_I & EQ5D_F & QALY & Total cost (£) & EQ5D_B & EQ5D_I & EQ5D_F & QALY & Total cost (£) \\
\hline Cluster 1 & 0.7491 & 0.7367 & 0.7967 & 0.7548 & 1753.33 & 0.7808 & 0.7208 & 0.6923 & 0.7287 & 1193.50 \\
\hline Cluster 2 & 0.7146 & 0.8083 & 0.8429 & 0.7935 & 900.98 & 0.6204 & 0.6903 & 0.7889 & 0.6975 & 1501.32 \\
\hline Cluster 3 & 0.7879 & 0.8394 & 0.9540 & 0.8552 & 1224.72 & 0.7254 & 0.6513 & 0.9223 & 0.7376 & 883.39 \\
\hline Cluster 4 & 0.6873 & 0.7346 & 0.7802 & 0.7342 & 1187.22 & 0.7075 & 0.7526 & 0.7393 & 0.7380 & 1329.61 \\
\hline Cluster 5 & 0.7355 & 0.7365 & 0.7134 & 0.7305 & 1422.09 & 0.8528 & 0.7888 & 0.9593 & 0.8474 & 1886.88 \\
\hline Cluster 6 & 0.8043 & 0.8145 & 0.8528 & 0.8215 & 2184.66 & 0.6585 & 0.6944 & 0.7178 & 0.6913 & 2189.66 \\
\hline Cluster 7 & 0.7656 & 0.7821 & 0.7323 & 0.7655 & 693.08 & 0.7404 & 0.7279 & 0.7963 & 0.7481 & 2420.59 \\
\hline Cluster 8 & 0.8599 & 0.8112 & 0.8104 & 0.8232 & 548.67 & \multicolumn{5}{|c|}{ No Cluster 8} \\
\hline
\end{tabular}

Costs are presented in GBP (£)

$A U C$ area under the curve, $E Q-5 D \_B$ EQ-5D at baseline, $E Q-5 D \_F$ EQ-5D at 12-month follow-up, $E Q-5 D \_I$ EQ-5D at 6-month follow-up, $P C I$ Patient Concerns Inventory, $Q A L Y$ quality-adjusted life-years 
an incremental cost of $£ 560$ and an incremental QALY of 0.03 , which then gave an ICER of $£ 21,449$. In both cases, the ICER value was less than the NICE threshold value of $£ 30,000$.

The cost-effectiveness analysis for all cluster (each row in each group) comparisons (see Appendix 2, Table 9 for all cluster results) shows that clusters 2, 3, 7 and 8 of the PCI intervention strategy were the dominant strategy against most clusters of the non-PCI cluster, except cluster 5 where the PCI might still be cost effective. For full results from the cluster analysis, see Appendix 2 Table 9.

To avoid repetition, only complete case scenarios were investigated in the sensitivity analysis.

\subsection{Sensitivity Analysis}

A bootstrap of 2000 values was simulated and plotted using a scatter plot as shown in Fig. 1. This scatter plot shows a higher density of bootstrapped cost-effectiveness ratios on the right-hand side of the chart but more on the south-east quadrant, which shows that the PCI intervention strategy has lower costs and more QALY values.

The CEAC presented in Fig. 2 shows lower probabilities of cost effectiveness at lower WTP thresholds and a higher probability of being cost effective at higher WTP thresholds. At a WTP threshold of $£ 0$ per QALY gained, the PCI intervention had a 0.61 probability of being cost effective; at a higher WTP threshold of $£ 20,000$ per QALY gained, the probability of being cost effective reached an approximate peak of 0.85 . This shows the PCI intervention is cost effective below the UK NICE threshold of $£ 20,000-30,000$.

From a WTP value of $£ 12,000$ per QALY gain, we observe a flattening of the curve such that the probability of being cost effective at $£ 12,000$ and the lower NICE threshold of $£ 20,000$ was almost equal, as shown with the dashed line in Fig. 2. For a WTP threshold range of $£ 0-20,000$ per QALY gained, the probability of being cost effective had a $95 \%$ CI of $0.80-0.83$.

\subsection{Results of the Qualitative Interviews with Consultants}

Three overarching themes were identified from the qualitative interviews with seven out of eight recruited consultants involved in the PCI intervention arm: (1) Enhancing the consultation - an integral holistic dimension, (2) utilisation of the PCI in routine care and (3) barriers and facilitators to implementation of PCI.

\subsubsection{Theme 1: Enhancing the Consultation—an Integral Holistic Dimension}

Consultants using the PCI reported an enhanced awareness of patients' overall post-treatment needs, presenting a more holistic view of the consultation.

"It's definitely an opportunity for the patients to talk about things they wouldn't normally discuss, and it also brought elements to the consultation to mind that I wouldn't have necessary enquired about." Participant 4

This promoted a shared understanding of the patient experience, especially surrounding emotional and psychosocial dimensions, with more ready offers or initiation of referrals to psychology and emotional support services. Use of the PCI also prompts and empowers patients to discuss sensitive topics such as intimacy and sexuality, which previously may
Fig. 1 Bootstrapping scatterplot for 2000 simulated values (complete case analysis)

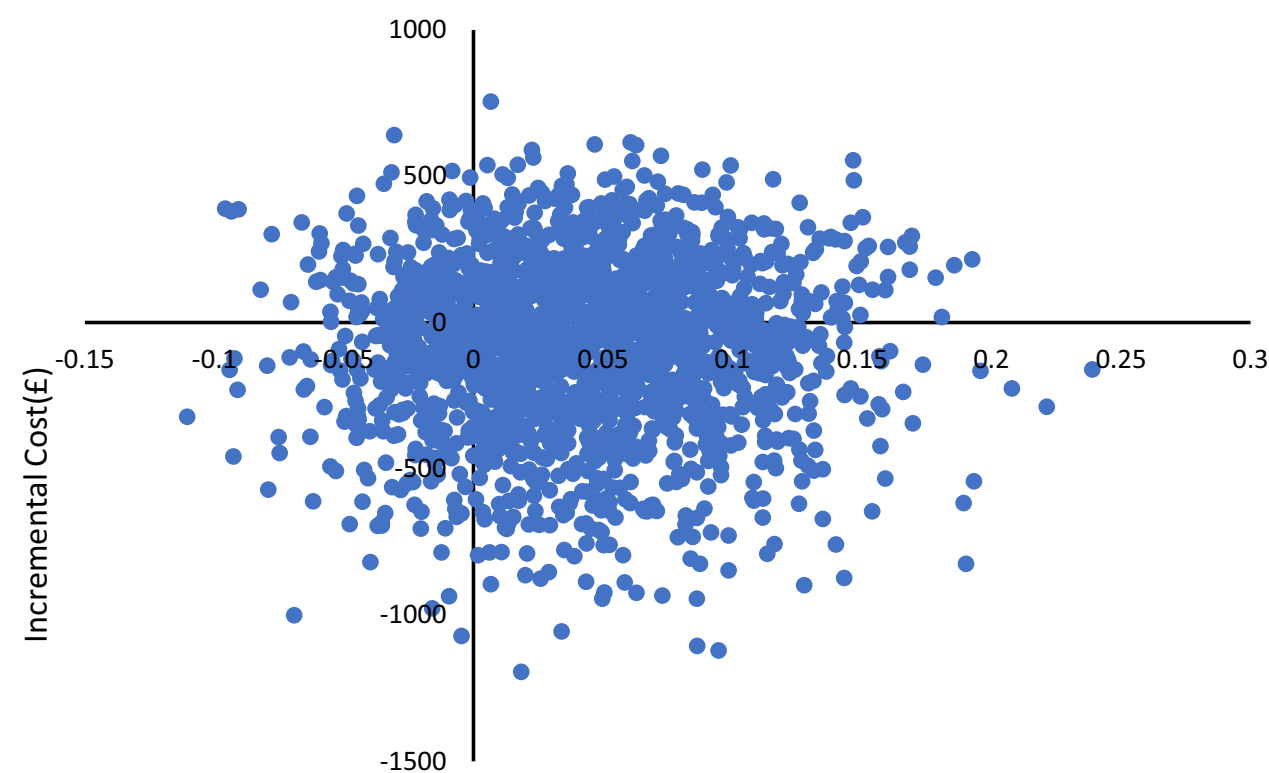

Incremental QALY 


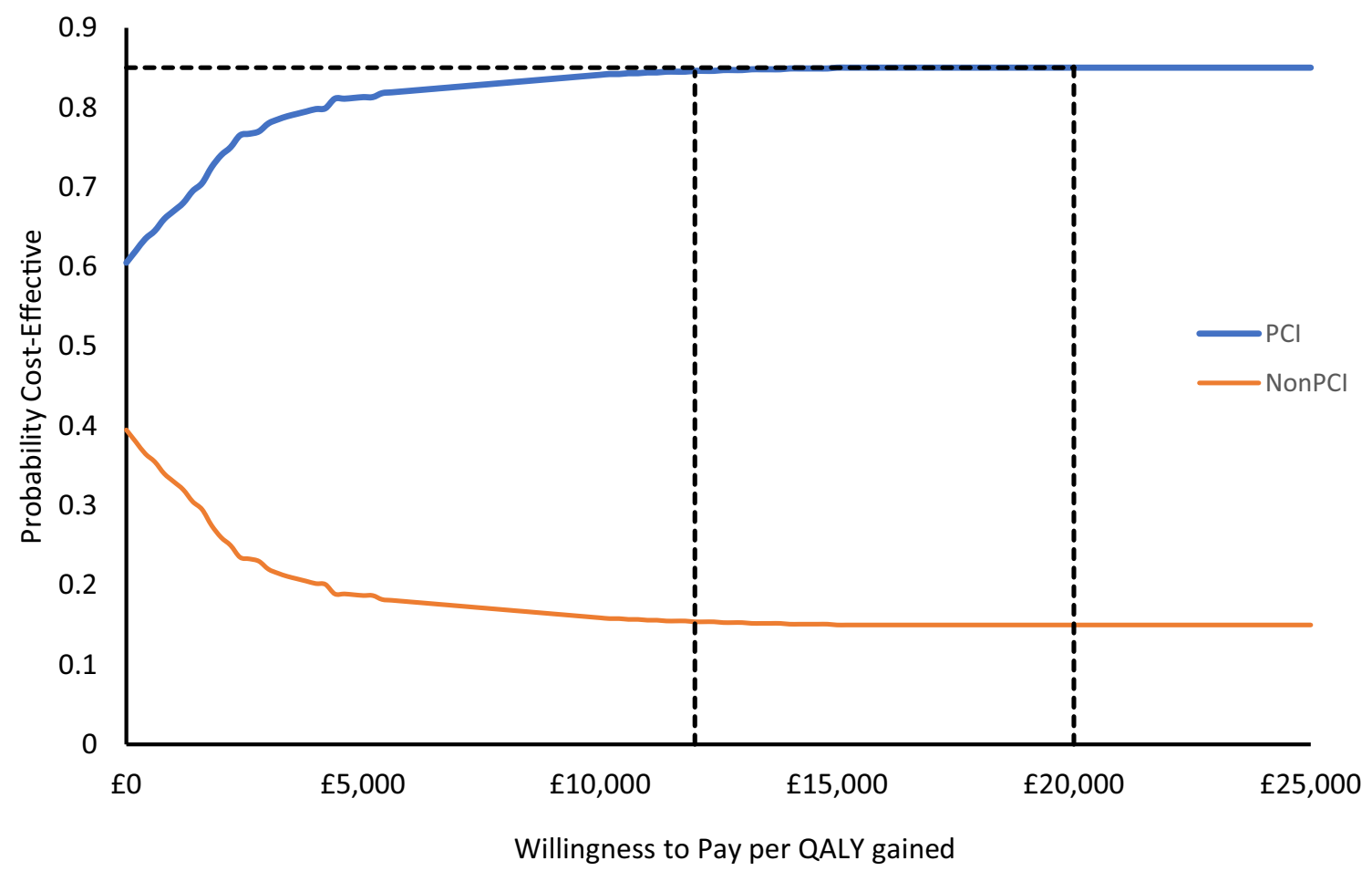

Fig. 2 The probability of the cost-effectiveness within the context of the willingness to pay threshold

have been considered irrelevant or not identified. Aligned with this was the clear acknowledgement of the discomfort around addressing sexuality, often with a diffusion of responsibility to other team members perceived as being better skilled to manage sensitive topics.

"The more sensitive things I think it is good for that because I suspect those things may well go unsaid or unasked." Participant 6

\subsubsection{Theme 2: Utilisation of $\mathrm{PCl}$ in Routine Care}

Utilisation of the PCI in routine care offers flexibility in its approach. How the PCI was integrated varied from clinician to clinician but also within their own scope of practice. For some, it framed the consultation from the onset; for others, it was used toward the end to focus on items not already addressed. Some participants incorporated a discussion of items throughout the consultation, whereas others varied their approach according to the items that were selected and were thus driven by the patient's needs.

\subsubsection{Theme 3: Barriers and Facilitators to Implementation of the $\mathrm{PCl}$}

Some consultants reported being sceptical at the onset of the trial, with the pervading thought that integration of the PCI would lengthen consultation time. This dissipated quickly once the PCI was in use, with consultants reporting that implementation enabled streamlining of consultations by directing the focus towards prioritisation of important items as indicated by the patient. As confidence in using the PCI developed, which was reported as happening quite rapidly, consultants reflected that patient concerns were more efficiently and productively managed during the consultation.

Consultants working within a well-established multidisciplinary team recognised the complement of skills needed to deliver high-quality patient-centred care and appeared more enthusiastic about the value of the PCI. In contrast, consultants that considered it their responsibility to 'fix' identified concerns or have an actionable solution identified this as a potential barrier to implementation of the PCI when no tangible solutions seemed within their reach.

"I am not in dread of any of them because ultimately you see yourself as a signpost in which very often you can send them to someone who can deal with it whether that be the emotional support or nurse specialist or wherever." Participant 6

Facilitators for the implementation of the PCI in routine clinical care included a one-off focused tailored training event $(<1 \mathrm{~h})$ at commencement, with no supplementary sessions deemed necessary. Important aspects embedded into training should be context setting, familiarisation 
with the tool, process of integrating into practice, advice and guidance on managing selection of multiple items, and dealing with more sensitive issues around intimacy, sexuality and fear of recurrence. Another facilitator was having adequate IT infrastructure for completion of PCI in advance of consultation, so that the flow of the consultation was not interrupted.

\section{Discussion}

HRQoL and survivorship are growing areas of interest within cancer follow-up care [31]. Our economic evaluation of the PCI intervention investigated the cost effectiveness of the PCI compared with not using the PCI in relation to consultations with cancer consultants, and our qualitative analysis provided insights from the consultants who used the PCI with their patients.

From Tables 5 and 6 , it is observed that the AUC in the PCI groups had higher values than the non-PCI groups but with the cost it was uncertain. All analyses conducted applying data imputation and complete case scenario showed similar results.

In Table 7, it is observed that the PCI group had a higher QALY value of 0.79 , whereas the non-PCI group had a lower QALY value of 0.76 . This provides evidence that the patients in the intervention group, on average, gained 0.03 QALYs per year. The results of the base-case analysis showed that the PCI group was, for most clusters, the most cost-effective or cost-saving intervention strategy. In general, the cost of the PCI group was lower by £295.91, showing that the NHS will be saving resources and funds if this strategy is implemented. This saving is due to more focused service use, as patients are given the exact consultations/treatments required. This result was further supported by the sensitivity analysis. The ICER plot showed a higher density of scatter plot in the south-east quadrant, indicating lower costs and higher effectiveness. The CEAC showed that no single intervention has $100 \%$ probability of being cost effective, but the PCI intervention has a higher probability of being cost effective at all willingness-to-pay thresholds.

The HNC PCI provided an effective means to conduct healthcare consultations by avoiding unnecessary NHS

Table 7 The average area under (QALY) the curve (EQ-5D-5L) of the PCI group vs. the non-PCI group

\begin{tabular}{lll}
\hline Area under the curve & PCI & Non-PCI \\
\hline Baseline to intermediate follow-up & 0.39 & 0.38 \\
Intermediate to 12-month follow-up & 0.40 & 0.38 \\
Total area under the curve & 0.79 & 0.76 \\
\hline
\end{tabular}

$P C I$ Patient Concerns Inventory, $Q A L Y$ quality-adjusted life-years costs by facilitating patients to identify their main points of concern and addressing those. The PCI intervention falls within the NICE guideline threshold of $£ 20,000$ per QALY gain [32].

The variation in costs in the different clusters observed in Tables 5 and 6 can be accounted for by the fewer visits to various consultants and the severity of the HNC. Certain treatment costs are higher than others, but patients getting the exact treatment or consultations needed minimises the costs associated with the health pathway. This elimination in costs from the treatment pathway is a major reason for the PCI intervention being cost saving in the overall analysis (see Table 2).

The benefit of including the PCI in HNC post-treatment consultations lies in its ability to focus the consultation to ensure it is holistic and tailored to individual patient-related issues compared with not using the PCI. Alongside cancerand treatment-related issues, mood and anxiety issues can also be picked up and addressed [33]. The findings are generalisable to other NHS settings in the UK, as the treatment methods and follow-up procedures are similar throughout the UK NHS.

We scoped the literature for similar studies in this area and only found one to be relevant. We found a cost-effectiveness study looking into the efficiency of a one-stop neck lump clinic in New Zealand [34]. However, it is not possible to compare the cost of this PCI intervention with similar intervention costs as there are no similar health economics studies investigating the cost of an inventory intervention to facilitate $\mathrm{HNC}$ care.

As evidenced by the qualitative interviews, consultants involved in this study were in favour of using the PCI within their clinical consultations in the future. The use of the PCI did not elongate consultation time but did enable clinicians to be more cognisant of the overall breadth of patients' needs, especially surrounding emotional and sexual wellbeing. The PCI can function as a trigger for patient issues, potentially allowing for more effective communication and subsequent earlier offers of advice, guidance or referral to the most appropriate team member or service. These qualitative data added depth to the standard analysis by highlighting issues not addressed by the main outcome measures [20].

Future studies should encapsulate the clusters according to the level of severity of the HNC in patients to help consolidate this PCI study. The inclusion of other costs, such as out-of-pocket costs, and use of the societal perspective could be further investigated. Researchers could translate and linguistically validate the available PCI in $\mathrm{HNC}$ into languages other than English and undertake cross-cultural studies regarding its utility.

\subsection{Strengths and Limitations}

Several studies have been conducted to investigate the effectiveness of the PCI in clinical consultations, but this is the 
first to include an economic evaluation component. Adding this economic evaluation analysis adds to the literature by bringing a different perspective to PCI intervention. The low cost of this PCI intervention for HNC care had a positive impact, with beneficial cost savings. In this study, the criteria for the cluster RCT ensured consultant contamination was avoided as this limited the chances of PCI or non-PCI sceptic consultants dominating the PCI or non-PCI group.

Most observed costs were linked to consultant time as the frequency of other resource use was low in the follow-up period. The number of patients with incomplete EQ-5D-5L data was high, which may have affected the final analysis, though the data imputation states otherwise. The PCI RCT follow-up consultations had to stop earlier than scheduled because of the coronavirus disease 2019 (COVID-19) global pandemic. In the future, post pandemic, it would be prudent to re-do this study as the NHS and other healthcare bodies have adopted increased non-face-to-face consultations and treatments. It would also be prudent to extend the study to more sites within the UK.

\section{Conclusion}

This is the first economic evaluation of a PCI in any setting and specifically in $\mathrm{HNC}$ care. The low cost of this PCI intervention for $\mathrm{HNC}$ care will make it an intervention of interest to NHS Healthcare Trusts in the UK and globally, especially as there was no significant difference in the mean consultation time between PCI and non-PCI groups [35]. Modest training requirements for health professionals is a small required cost, but the $\mathrm{PCI}$ intervention appears to generate a cost-effective benefit to patients from an NHS perspective if rolled out as part of routine $\mathrm{HNC}$ care.

The HNC PCI provided an effective means to conduct the healthcare consultations by avoiding unnecessary NHS costs by identifying the main points of concern and addressing those. The PCI intervention falls within the NICE guideline threshold of $£ 20,000$ per QALY gain; however, it is based on very small incremental differences in cost and effect between groups. For this reason, qualitative evidence was also used to expand the interpretation of the economic evaluation and findings.

\section{Appendix 1}

Main results from the clinical effectiveness paper Rogers et al. (2020) [16]

Journal Name: European Archives of Oto-Rhino-Laryngology

Article Title: Improving quality of life through the routine use of the patient concerns inventory for head and neck cancer patients: baseline results in a cluster preference randomised controlled trial

Authors: Simon N. Rogers, Christine Allmark, Fazilet Bekiroglu, Rhiannon Tudor Edwards, Gillon Fabbroni, Robert Flavel, Victoria Highet, Michael W. S. Ho, Gerald M. Humphris, Terry M. Jones, Owais Khattak, Jeffrey Lancaster, Christopher Loh, Derek Lowe, Cher Lowies, Dominic Macareavy, James Moor, T. K. Ong, A. Prasai, Nicholas Roland, Cherith Semple, Llinos Haf Spencer, Sank Tandon, Steven J. Thomas, Andrew Schache, Richard J. Shaw \& Anastasios Kanatas

Affiliation and email corresponding author: Simon N. Rogers.

Faculty of Health and Social Care, Edge Hill University, Ormskirk, L39 4QP, UK.

Liverpool Head and Neck Centre, Liverpool University Hospital Aintree, Liverpool, UK.

Contact Simon N. Rogers SIMONN.ROGERS@liverpoolft.nhs.uk.

Conflict of interest:

We do not have financial disclosure or any conflict of interest.

Result from paper:

From 511 patients first identified as eligible when screening for the multi-disciplinary tumour board meetings, 288 attended a first routine outpatient baseline study clinic after completion of their treatment, median (IQR) of 103 (71-162) days.

At baseline, the two trial groups were similar in demographic and clinical characteristics as well as in HealthRelated Quality of Life (HRQOL) measures apart from differences in tumour location, tumour staging and mode of treatment. Overall quality of life (QOL) was outstanding for 6\% (18), very good for 30\% (87), good for 33\% (94), fair for $22 \%$ (63), poor for 6\% (17) and very poor for 3\% (9); i.e. $31 \%(89)$ had overall QOL that was less than good and $69 \%$ (199) had overall QOL that was good or better. Also, $45 \%$ (129) stated Distress Ther- mometer values of $\geq 4$ and the median (IQR) UWQOL emotional subscale score was 75 (59-88). Dysfunction in regard to UWQOL items were notably higher in regard to salivation $(34 \%, 99)$, pain $(29 \%$, $83)$, fears of recurrence $(26 \%, 75)$, and taste $(20 \%, 57)$.

Using the PCI in routine post-treatment head and neck cancer clinics do not elongate consultation time. Consultation times were similar between the two groups indicating that the use of the PCI did not appear to have impacted the timetabling of clinic sessions.

\section{Appendix 2}

See Tables 8 and 9. 
Table 8 Incremental cost-effectiveness of PCI intervention cluster in comparison to Non-PCI cluster

\begin{tabular}{|c|c|c|c|c|c|}
\hline \multirow[t]{2}{*}{ Intervention } & \multirow[t]{2}{*}{ Main cost } & \multirow[t]{2}{*}{ QALYs } & \multicolumn{2}{|c|}{ Incremental } & \multirow[t]{2}{*}{ ICER } \\
\hline & & & Cost & QALYs & \\
\hline \multicolumn{6}{|c|}{ From Table 3 (Row 1) } \\
\hline PCI & $£ 1,753$ & 0.76 & $£ 390$ & 0.03 & 14215 \\
\hline Non-PCI & $£ 1,364$ & 0.73 & & & \\
\hline \multicolumn{6}{|c|}{ From table 4 (Row 1) } \\
\hline PCI & $£ 1,753$ & 0.76 & $£ 560$ & 0.03 & 21449 \\
\hline Non-PCI & $£ 1,194$ & 0.73 & & & \\
\hline
\end{tabular}

Table 9 Incremental cost-effectiveness of PCI intervention strategy in comparison to non-PCI intervention for each cluster for complete and inputted data

\begin{tabular}{|c|c|c|c|c|c|c|}
\hline \multirow[b]{2}{*}{ Non-PCI Cluster } & \multicolumn{3}{|c|}{ Complete case scenario } & \multicolumn{3}{|l|}{ Inputted data } \\
\hline & Inc. Cost & Inc. Effect & ICER(Cost/QALY) & Inc. Cost & Inc. Effect & ICER(Cost/QALY) \\
\hline & PCI Cluster 1 & & & PCI Cluster 1 & & \\
\hline Cluster 1 & 559.83 & 0.03 & 21428.46 & 389.49 & 0.03 & 14231.21 \\
\hline Cluster 2 & 252.01 & 0.06 & 4398.66 & -417.44 & -0.02 & 18111.04 \\
\hline Cluster 3 & 869.95 & 0.02 & 50521.16 & 811.72 & -0.02 & Dominated \\
\hline Cluster 4 & 423.72 & 0.02 & 25255.54 & 142.34 & -0.01 & Dominated \\
\hline Cluster 5 & -133.55 & -0.09 & 1441.31 & 253.17 & -0.15 & Dominated \\
\hline Cluster 6 & -436.33 & 0.06 & Dominant & -243.40 & 0.03 & Dominant \\
\hline \multirow[t]{2}{*}{ Cluster 7} & -667.26 & 0.01 & Dominant & -916.07 & 0.00 & 531910.85 \\
\hline & PCI Cluster 2 & & & PCI Cluster 2 & & \\
\hline Cluster 1 & -292.53 & 0.06 & Dominant & -47.39 & 0.14 & Dominant \\
\hline Cluster 2 & -600.34 & 0.10 & Dominant & -854.32 & 0.09 & Dominant \\
\hline Cluster 3 & 17.59 & 0.06 & 314.44 & 374.84 & 0.09 & 4052.34 \\
\hline Cluster 4 & -428.64 & 0.06 & Dominant & -294.54 & 0.10 & Dominant \\
\hline Cluster 5 & -985.91 & -0.05 & 18278.70 & -183.71 & -0.04 & 4453.53 \\
\hline Cluster 6 & -1288.68 & 0.10 & Dominant & -680.28 & 0.14 & Dominant \\
\hline \multirow[t]{2}{*}{ Cluster 7} & -1519.62 & 0.05 & Dominant & -1352.95 & 0.11 & Dominant \\
\hline & PCI Cluster 3 & & & PCI Cluster 3 & & \\
\hline Cluster 1 & 31.22 & 0.13 & 246.77 & 330.39 & 0.16 & 2118.34 \\
\hline Cluster 2 & -276.60 & 0.16 & Dominant & -476.55 & 0.11 & Dominant \\
\hline Cluster 3 & 341.34 & 0.12 & 2902.08 & 752.62 & 0.11 & 6697.38 \\
\hline Cluster 4 & -104.89 & 0.12 & Dominant & 83.24 & 0.12 & 683.89 \\
\hline Cluster 5 & -662.16 & 0.01 & Dominant & 194.07 & -0.02 & Dominated \\
\hline Cluster 6 & -964.94 & 0.16 & Dominant & -302.50 & 0.16 & Dominant \\
\hline \multirow[t]{2}{*}{ Cluster 7} & -1195.87 & 0.11 & Dominant & -975.17 & 0.13 & Dominant \\
\hline & PCI Cluster 4 & & & PCI Cluster 4 & & \\
\hline Cluster 1 & -6.28 & 0.01 & Dominant & 55.89 & 0.04 & 1553.42 \\
\hline Cluster 2 & -314.10 & 0.04 & Dominant & -751.04 & -0.01 & 52018.28 \\
\hline Cluster 3 & 303.84 & 0.00 & Dominated & 478.12 & -0.01 & Dominated \\
\hline Cluster 4 & -142.39 & 0.00 & 37153.67 & -191.26 & 0.00 & Dominant \\
\hline Cluster 5 & -699.66 & -0.11 & 6176.94 & -80.43 & -0.14 & 568.95 \\
\hline Cluster 6 & -1002.44 & 0.04 & Dominant & -577.00 & 0.04 & Dominant \\
\hline \multirow[t]{2}{*}{ Cluster 7} & -1233.37 & -0.01 & 88312.27 & -1249.67 & 0.01 & Dominant \\
\hline & PCI Cluster 5 & & & PCI Cluster 5 & & \\
\hline Cluster 1 & 228.58 & 0.00 & 125841.16 & 480.17 & 0.02 & 20103.20 \\
\hline Cluster 2 & -79.23 & 0.03 & Dominant & -326.77 & -0.03 & 12315.62 \\
\hline
\end{tabular}


Table 9 (continued)

\begin{tabular}{|c|c|c|c|c|c|c|}
\hline \multirow[b]{2}{*}{ Cluster 3} & \multicolumn{3}{|c|}{ Complete case scenario } & \multicolumn{3}{|c|}{ Inputted data } \\
\hline & 538.70 & -0.01 & Dominated & 902.39 & -0.02 & Dominated \\
\hline Cluster 4 & 92.48 & -0.01 & Dominated & 233.01 & -0.01 & Dominated \\
\hline Cluster 5 & -464.80 & -0.12 & 3973.68 & 343.85 & -0.15 & Dominated \\
\hline Cluster 6 & -767.57 & 0.04 & Dominant & -152.73 & 0.03 & Dominant \\
\hline \multirow[t]{2}{*}{ Cluster 7} & -998.51 & -0.02 & 56523.32 & -825.39 & -0.01 & 158550.01 \\
\hline & \multicolumn{3}{|l|}{ PCI Cluster 6} & \multicolumn{3}{|c|}{ PCI Cluster 6} \\
\hline Cluster 1 & 991.16 & 0.09 & 10669.61 & 1089.68 & 0.10 & 11392.51 \\
\hline Cluster 2 & 683.34 & 0.12 & 5508.01 & 282.74 & 0.05 & 6251.14 \\
\hline Cluster 3 & 1301.27 & 0.08 & 15493.34 & 1511.91 & 0.05 & 29042.91 \\
\hline Cluster 4 & 855.05 & 0.08 & 10234.32 & 842.53 & 0.06 & 13723.38 \\
\hline Cluster 5 & 297.77 & -0.03 & Dominated & 953.36 & -0.08 & Dominated \\
\hline Cluster 6 & -5.00 & 0.13 & Dominant & 456.79 & 0.10 & 4539.23 \\
\hline \multirow[t]{2}{*}{ Cluster 7} & -235.93 & 0.07 & Dominant & -215.88 & 0.07 & Dominant \\
\hline & \multicolumn{3}{|l|}{ PCI Cluster 7} & \multicolumn{3}{|c|}{ PCI Cluster 7} \\
\hline Cluster 1 & -500.43 & 0.04 & Dominant & -533.72 & 0.02 & Dominant \\
\hline Cluster 2 & -808.24 & 0.07 & Dominant & -1340.66 & -0.03 & 42363.74 \\
\hline Cluster 3 & -190.31 & 0.03 & Dominant & -111.50 & -0.02 & 4492.27 \\
\hline Cluster 4 & -636.54 & 0.03 & Dominant & -780.88 & -0.02 & 50432.10 \\
\hline Cluster 5 & -1193.81 & -0.08 & 14574.50 & -670.04 & -0.16 & 4225.56 \\
\hline Cluster 6 & -1496.58 & 0.07 & Dominant & -1166.62 & 0.02 & Dominant \\
\hline \multirow[t]{2}{*}{ Cluster 7} & -1727.52 & 0.02 & Dominant & -1839.28 & -0.01 & 178234.70 \\
\hline & \multicolumn{3}{|l|}{ PCI Cluster 8} & \multicolumn{3}{|c|}{ PCI Cluster 8} \\
\hline Cluster 1 & -644.83 & 0.09 & Dominant & -698.88 & 0.03 & Dominant \\
\hline Cluster 2 & -952.65 & 0.13 & Dominant & -1505.81 & -0.02 & 72128.07 \\
\hline Cluster 3 & -334.72 & 0.09 & Dominant & -276.65 & -0.01 & 19690.34 \\
\hline Cluster 4 & -780.94 & 0.09 & Dominant & -946.03 & 0.00 & 200673.21 \\
\hline Cluster 5 & -1338.21 & -0.02 & 55160.36 & -835.20 & -0.15 & 5650.87 \\
\hline Cluster 6 & -1640.99 & 0.13 & Dominant & -1331.77 & 0.03 & Dominant \\
\hline Cluster 7 & -1871.92 & 0.08 & Dominant & -2004.44 & 0.00 & Dominant \\
\hline
\end{tabular}

Supplementary Information The online version contains supplementary material available at https://doi.org/10.1007/s41669-021-00320-4.

Acknowledgements The authors acknowledge the collaborations between NHS sites with respect to the intellectual property related to the PCI trial. We also thank the patients who participated and the public and patient involvement representatives for their support in submitting the grant application and for their involvement on the steering group. Our thanks also to Dr Catherine Lawrence, reader support for Professor Rhiannon Tudor Edwards. Health and Care Economics Cymru is funded by Welsh Government through Health and Care Research Wales.

Funding This trial is funded by the RfPB on behalf of the NIHR (PBPG-0215-36047). This paper presents independent research funded by the National Institute for Health Research (NIHR) under its Research for Patient Benefit (RfPB) Programme (Grant Reference Number PB-PG-0215-36047). The views expressed are those of the authors and not necessarily those of the NHS, the NIHR or the Department of Health.

\section{Declarations}

Funding This paper presents independent research funded by the National Institute for Health Research (NIHR) under its Research for Patient Benefit (RfPB) Programme (Grant Reference Number PB-PG-0215-36047). The funder was not involved in the design of the study and collection, analysis, and interpretation of data, or in writing the manuscript. The views and opinions expressed are those of the author(s) and not necessarily those of the NIHR or the Department of Health and Social Care.

Ethical approval The Patient Concerns Inventory study received ethical approval from North West-Liverpool Central Research Ethics Committee, REC reference: IRAS 16/NW/0465. Project ID: 189554. It also had approval from the Health Research Authority (HRA). The Research and Development Department at Aintree Hospital NHS Trust (AUH) coordinated the trial and AUH was the sponsor for the trial.

Conflict of interest The authors declare that they have no known competing financial interests or personal relationships that could have appeared to influence the work reported in this paper. 
Author contributions STY and RTE reviewed the Health Economic Analysis Plan (HEAP). LHS and RTE drafted initial manuscript and VE undertook the health economics analysis. VE, LHS and RTE wrote the health economics paper; CJS and JRH carried out the qualitative analysis from the consultant interviews; SNR and DL conceptualised the trial and designed and wrote the study protocol; SNR and DL contributed to the advanced health economics paper. AK, CJS, DL, LHS, SNR, STY, RTE were Steering Group members and were responsible for the delivery for the trial. All authors read and approved the final manuscript.

Consent for publication This paper presents independent research funded by the National Institute for Health Research (NIHR). The views expressed are those of the author(s) and not necessarily those of the NHS, the NIHR or the Department of Health and Social Care.

Consent for participate As part of the trail patients consented to the study.

Availability of data and material The datasets used and/or analysed during the current study are available from the corresponding author on reasonable request or Please contact the Principal Investigator Simon Rogers for datasets used in the PCI study.

Code availability The code for the PCI analysis used in this study is available from the corresponding author upon request.

Open Access This article is licensed under a Creative Commons Attribution-NonCommercial 4.0 International License, which permits any non-commercial use, sharing, adaptation, distribution and reproduction in any medium or format, as long as you give appropriate credit to the original author(s) and the source, provide a link to the Creative Commons licence, and indicate if changes were made. The images or other third party material in this article are included in the article's Creative Commons licence, unless indicated otherwise in a credit line to the material. If material is not included in the article's Creative Commons licence and your intended use is not permitted by statutory regulation or exceeds the permitted use, you will need to obtain permission directly from the copyright holder. To view a copy of this licence, visit http://creativecommons.org/licenses/by-nc/4.0/.

\section{References}

1. National Health Service (NHS). Head and neck cancer-NHS. 2020. https://www.nhs.uk/conditions/head-and-neck-cancer/. Cited 10 Nov 2020

2. Devins GM, Wong JC, Payne AYM, Lebel S, Lee RNF, Mah K, Irish J, Rodin G. Distancing, self-esteem, and subjective well-being in head and neck cancer. Psychooncology. 2015;24(11):1506-13

3. Griffin SJ, Kinmonth AL, Veltman MWM, Gillard S, Grant J, Stewart M. Effect on health-related outcomes of interventions to alter the interaction between patients and practitioners: a systematic review of trials. Ann Fam Med. 2004;2(6):595-608.

4. Rogers SN, Clifford N, Lowe D. Patient and carer unmet needs: a survey of the British association of head and neck oncology nurses. Br J Oral Maxillofac Surg. 2011;49(5):343-8.

5. Miller N, Rogers SN. A review of question prompt lists used in the oncology setting with comparison to the Patient Concerns Inventory. Eur J Cancer Care (Engl). 2016;27(1):1-15.

6. Brown RF, Butow PN, Dunn SM, Tattersall MHN. Promoting patient participation and shortening cancer consultations: a randomised trial. Br J Cancer. 2001;85(9):1273-9.
7. Ghazali N, Roe B, Lowe D, Rogers SN. Uncovering patients' concerns in routine head and neck oncology follow up clinics: an exploratory study. Br J Oral Maxillofac Surg. 2013;51(4):294300. https://doi.org/10.1016/j.bjoms.2012.08.002.

8. Kanatas A, Humphris G, Lowe D, Rogers SN. Further analysis of the emotional consequences of head and neck cancer as reflected by the Patients' Concerns Inventory. Br J Oral Maxillofac Surg. 2015;53(8):711-8. https://doi.org/10.1016/j.bjoms.2015.02.026.

9. Rogers SN, Lowe D. Screening for dysfunction to promote multidisciplinary intervention by using the university of washington quality of life questionnaire. Arch Otolaryngol Head Neck Surg. 2009;135(4):369-75.

10. Shunmugasundaram C, Rutherford C, Butow PN, Sundaresan $\mathrm{P}$, Dhillon HM. Content comparison of unmet needs self-report measures used in patients with head and neck cancer: a systematic review. Psychooncology. 2019;28(12):2295-306.

11. Rogers SN, Lowe D, Lowies C, Yeo ST, Allmark C, Mcavery D, Humphris GM, Flavel R, Semple C, Thomas SJ, Kanatas A. Improving quality of life through the routine use of the patient concerns inventory for head and neck cancer patients: a cluster preference randomized controlled trial. BMC Cancer. 2018;18(1):1-10.

12. Rogers SN, Allmark C, Bekiroglu F, Edwards RT, Fabbroni G, Flavel R, Highet V, Ho MWS, Humphris GM, Jones TM, Khattak O, Lancaster J, Loh C, Lowe D, Lowies C, Macareavy D, Moor J, Ong TK, Prasai A, Roland N, Semple C, Spencer LH, Tandon S, Thomas SJ, Schache A, Shaw RJ, Kanatas A. Improving quality of life through the routine use of the patient concerns inventory for head and neck cancer patients: main results of a cluster preference randomised controlled trial. Eur Arch Oto Rhino Laryngol. 2021;278(9):3435-49. https://doi.org/10.1007/ s00405-020-06533-3.

13. Rogers SN, Lowe D. An evaluation of the head and neck cancer patient concerns inventory across the merseyside and cheshire network. Br J Oral Maxillofac Surg. 2014;52(7):615-23. https:// doi.org/10.1016/j.bjoms.2014.04.011.

14. Richardson A, Dewar S, Barnish L, Tritton J, Archer J, Corrigan A, Dawson T, Young N, Ainsworth K, Brennan J. Holistic Needs Assessment for people with cancer. A practical guide for healthcare professionals. National Cancer Action Team. 2017. http:// www.gloshospitals.nhs.uk/SharePoint58/HealthPsychologyWebD ocuments/The_holistic_needs_assessment_for_people_with_cancer_A_practical_Guide_NCAT.pdf

15. Richards M, Corner J, Maher J. The National Cancer Survivorship Initiative: new and emerging evidence on the ongoing needs of cancer survivors. Br J Cancer. 2011;105(S1):S1-4. https://doi.org/ 10.1038/bjc.2011.416.

16. Rogers SN, Allmark C, Bekiroglu F, Tudor R, Gillon E. Improving quality of life through the routine use of the patient concerns inventory for head and neck cancer patients: baseline results in a cluster preference randomised controlled trial. Eur Arch OtoRhino-Laryngol. 2020;277(12):3435-47. https://doi.org/10.1007/ s00405-020-06077-6.

17. Ridyard $\mathrm{CH}$, Hughes DA. Taxonomy for methods for resource use measurement. Health Econ. 2015;24:372-8.

18. Herdman M, Gudex C, Lloyd A, Janssen M, Kind P, Parkin D, Bonsel G, Badia X. Development and preliminary testing of the new five-level version of EQ-5D (EQ-5D-5L). Qual Life Res. 2011;20(10):1727-36.

19. Sutton EJ, Coast J. Development of a supportive care measure for economic evaluation of end-of-life care using qualitative methods. Palliat Med. 2013;28(2):151-7.

20. Coast J. Qualitative methods for health economics. London: Rowman \& Littlefield International Ltd; 2017. 
21. Clarke V, Braun V. Thematic analysis. J Posit Psychol. 2017;12(3):297-8. https://doi.org/10.1080/17439760.2016.12626 13.

22. Rakićević J, Savić G, Bulajić M. Selecting an appropriate method for missing data imputation: A case of countries ranking. In Symposium proceedings-XV International symposium Symorg 2016: Reshaping the Future Through Sustainable Business Development and Entepreneurship. University of Belgrade, Faculty of Organizational Sciences; 2016.

23. Roberts MB, Sullivan MC, Winchester SB. Examining solutions to missing data in longitudinal nursing research. J Spec Pediatr Nurs. 2017;22(2):12179. https://doi.org/10.1111/jspn.12179.

24. Curtis L, Burns A. Unit costs of health and social care 2020. Personal Social Services Research Unit. Personal Social Services Research Unit, Kent, UK; 2020.

25. NHS Improvement. National Cost Collection Data. 2020 [Cited 2020 Apr 21]. https://improvement.nhs.uk/resources/nationalcost-collection/

26. Charles JM, Edwards RT, Bywater T, Hutchings J. Micro-costing in public health economics: steps towards a standardized framework, using the incredible years toddler parenting program as a worked example. Prev Sci. 2013;14(4):377-89.

27. Van Hout B, Janssen MF, Feng Y, Kohlmann T, Busschbach J. Interim scoring for the EQ-5D-5L: mapping the EQ-5D-5L to EQ-5D-3L. Value Health. 2012;15(5):708-15. https://doi.org/10. 1016/j.jval.2012.02.008.

28. National Institute for Health and Care Excellence. Position statement on use of the EQ-5D-5L value set for England (updated October 2019). 2019. https://www.nice.org.uk/about/what-we-do/ our-programmes/nice-guidance/technology-appraisal-guidance/ eq-5d-51

29. Barber JA, Thompson SG. Analysis of cost data in randomized trials: an application of the non-parametric bootstrap. Stat Med. 2000;19(23):3219-36.

30. Xu X, Xia L, Zhang Q, Wu S, Wu M, Liu H. The ability of different imputation methods for missing values in mental measurement questionnaires. BMC Med Res Methodol. 2020;20:1-9.

31. Morrison V, Spencer LH, Totton N, Pye K, Yeo ST, Butterworth C, Hall L, Whitaker R, Edwards RT, Timmis LJ, Hoare Z, Neal RD, Wilkinson C, Leeson S. Trial of optimal personalised care after treatment-gynaecological cancer (TOPCAT-G). Int J Gynecol Cancer. 2018;28(2):401-11. http://insights.ovid.com/ crossref?an $=00009577-201802000-00028$

32. Appleby J, Devlin N, Parkin D. NICE's cost-effectiveness threshold. BMJ. 2007;335:358-9. https://doi.org/10.1136/bmj.39308. 560069.BE.

33. Kanatas A, Ghazali N, Lowe D, Rogers SN. The identification of mood and anxiety concerns using the patients concerns inventory following head and neck cancer. Int J Oral Maxillofac Surg. 2012;41(4):429-36.

34. Linkhorn H, Pandya H, Ramsaroop R, Wade M, Smith K. Neck lump clinic: a new initiative at North Shore Hospital. ANZ J Surg. 2019;89(7):853-7.

35. Rogers SN, Semple C, Humphris G, Lowe D, Kanatas A. Using a patient prompt list to raise concerns in oncology clinics does not necessarily lead to longer consultations. Br J Oral Maxillofac Surg. 2020;58(9):1164-71. https://doi.org/10.1016/j.bjoms.2020. 08.035 .

\section{Authors and Affiliations}

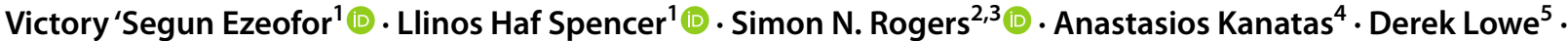 Cherith J. Semple ${ }^{6,7}$. Jeffrey R. Hanna ${ }^{8} \cdot$ Seow Tien Yeo $^{1} \cdot$ Rhiannon Tudor Edwards $^{1}$}

Llinos Haf Spencer

1.spencer@bangor.ac.uk

Simon N. Rogers

SIMONN.ROGERS@liverpoolft.nhs.uk

Anastasios Kanatas

anastasios.kanatas@nhs.net

Derek Lowe

astraglobeltd@btconnect.com

Cherith J. Semple

cherith.semple@setrust.hscni.net

Jeffrey R. Hanna

jeffrey.hanna@psych.ox.ac.uk

Seow Tien Yeo

s.t.yeo@bangor.ac.uk

Rhiannon Tudor Edwards

r.t.edwards@bangor.ac.uk

1 Centre for Health Economics and Medicines Evaluation

(CHEME), School of Medical and Health Sciences,
Ardudwy Hall, Normal Site, Bangor University, Bangor, Wales LL57 2PZ, UK

2 Faculty of Health, Social Care and Medicine, Edge Hill University, Ormskirk L39 4QP, England

3 Liverpool Head and Neck Centre, Liverpool University Hospital Aintree, Liverpool, England, UK

4 Leeds Teaching Hospitals and St James Institute of Oncology, Leeds Dental Institute and Leeds General Infirmary, Leeds, England, UK

5 Astraglobe Ltd, 24 Trinity Place, Congleton, Cheshire, England CW12 3JB, UK

6 Institute of Nursing and Health Research, Ulster University, Belfast, Northen Ireland BT37 0QB, UK

7 South Eastern Health and Social Care Trust, Belfast, Northern Ireland BT16 1RH, UK

8 Department of Psychiatry, Warneford Hospital, University of Oxford, Oxford, England, UK 